\title{
Lidil
}

Revue de linguistique et de didactique des langues

\section{(Dé)construction de clôtures identitaires dans un espace scolaire : un regard sociolinguistique impliqué}

Patricia Lambert

\section{(2) OpenEdition \\ Journals}

Édition électronique

URL : http://journals.openedition.org/lidil/2736

DOI : $10.4000 /$ lidil.2736

ISSN : $1960-6052$

Éditeur

UGA Éditions/Université Grenoble Alpes

Édition imprimée

Date de publication : 1 mai 2009

Pagination : 43-56

ISBN : 978-2-84310-139-7

ISSN : 1146-6480

Référence électronique

Patricia Lambert, "(Dé)construction de clôtures identitaires dans un espace scolaire : un regard sociolinguistique impliqué », Lidil [En ligne], 39 | 2009, mis en ligne le 01 décembre 2010, consulté le 02 mai 2019. URL : http://journals.openedition.org/lidil/2736 ; DOI : 10.4000/lidil.2736 


\title{
(Dé)construction de clôtures identitaires dans un espace scolaire : un regard sociolinguistique impliqué
}

\author{
Patricia LAMBERT*
}

\begin{abstract}
RÉSUMÉ
Dans la continuité d'une série de travaux grenoblois sur les pratiques langagières de descendants de migrants, nous avons consacré une recherche sociolinguistique ethnographique à l'étude des répertoires plurilectaux d'élèves d'une classe de lycée professionnel.

Fondé sur cette recherche, ce texte propose une analyse interprétative de processus de différenciations sociolangagières féminines et de construction d'altérités radicales dans cette classe. L'analyse indique notamment que l'attribution d'une identité monostyle dépréciée tend à masquer l'étendue des ressources langagières de certaines jeunes filles.

L'observation de ces effets de masquage plaide tout particulièrement, d'un point de vue didactique, en faveur d'une intégration accrue de contenus sociolinguistiques dans la formation des enseignants.
\end{abstract}

\section{ABSTRACT}

In continuity with a series of previous studies undertaken by researchers at the University of Grenoble on language usage and practices of descendents of migrants, we have conducted an ethnographic and sociolinguistic study on the plurilectal repertoires of pupils at a professional secondary school.

Based on this study, the present text offers an interpretative analysis of the process of sociolinguistic differentiation observed in female subjects and the construction of radical otherness in this class. The analysis reveals that attributing pupils with a depreciative mono-style identity tends to mask the range of linguistic resources of certain of these young ladies. The exposure through the study of this masking effect is of direct relevance from a didactic point of vue, to integrating sociolinguistic content much more substantially in teacher education.

* UMR 5191 ICAR/INRP, Lidilem. 
Motivée par un questionnement sur les possibilités d'une meilleure prise en compte de la pluralité des ressources langagières et culturelles des élèves, une proposition de recherche-action-formation a été adressée en 2001 par la direction d'un lycée professionnel à la responsable du Lidilem ${ }^{1}$.

En réponse à cette proposition, une convention de recherche a été élaborée, définissant trois axes principaux d'activités : l'étude des répertoires plurilectaux des élèves; l'organisation de séminaires de formation en direction des enseignants de lettres/histoire et de lettres/ anglais ; la conception et l'expérimentation d'activités scolaires et de ressources pédagogiques. Cette recherche s'est déroulée pendant près de quatre ans et a comporté diverses productions de données, l'élaboration et la mise en œuvre de séquences pédagogiques en cours de français et d'anglais, ainsi que des actions de formation. La forte représentation d'élèves potentiellement plurilingues dans l'établissement, les jugements dépréciatifs à l'égard de la « façon de parler » de certains d'entre eux, ainsi que l'observation de phénomènes d'attributions négatives mutuelles, entre des groupes d'élèves et des enseignants, nous ont conduite à approfondir certaines des voies tracées par une didactique de la variation et du plurilinguisme (Billiez, 1998).

C'est dans ce cadre, et dans la continuité d'une série de travaux grenoblois sur les pratiques langagières de descendants de migrants, que j'ai consacré ma recherche doctorale à l'étude des répertoires plurilectaux ${ }^{2}$ des jeunes filles d'une classe de seconde professionnelle de bureautique (Lambert, 2005).

1. Jacqueline Billiez, directrice du Laboratoire de Linguistique et Didactique des Langues Étrangères et Maternelles, Grenoble 3. D'autres chercheurs de cette équipe ont participé sous diverses formes à cette recherche-action : Elisabeth Calaque, Marinette Matthey, Cyril Trimaille.

2. Dans le contexte étudié, la question de la pluralité linguistique et culturelle dépasse celle du plurilinguisme et invite à reprendre l'expression «plurilectalisme » proposée par des sociolinguistes rouennais (Leconte et Delabarre, 2003). 


\section{Différenciations sociolangagières et construction d'altérités radicales dans une classe de seconde professionnelle}

Ce groupe-classe est majoritairement composé de descendantes de migrants de milieu socio-économique défavorisé. Sur les 23 élèves (16 à 18 ans), 19 sont des jeunes filles, et à l'exception de l'une d'entre elles, toutes les adolescentes affirment vivre au contact régulier de plusieurs langues. Sans être exhaustive, la liste suivante de lectes déclarés ${ }^{3}$ par des sujets est à même de donner un aperçu de l'hétérogénéité constitutive de leurs répertoires : arabes maghrébins, arménien, « comorien », coratin, français, « français cassé », italien, kabyle, portugais, « remix », etc.

\section{Le groupe des 6 et le groupe des 13}

Tout au long de l'année scolaire, le réseau « classe » se caractérise par la construction de deux groupes de pairs féminins ${ }^{4}$, entendus comme deux mini-réseaux affinitaires que nous appellerons le groupe des 6 et le groupe des 13 sur la base du nombre de leurs membres respectifs.

Des différenciations entre ces groupes d'élèves se manifestent d'abord dans la manière dont le réseau relationnel de la classe est décrit par les différents acteurs - élèves et enseignants. Les deux groupes reçoivent en effet un ensemble de désignations qui opèrent une focalisation variable sur des traits scolaires, sociaux, personnels, ethniques et langagiers ${ }^{5}$ : « sages/sauvages » $($ ELs) « françaises/algériennes » $($ ELs $)$

3. Les citations de propos d'enquêtés sont mises en italique et entre guillemets dans le texte. Il s'agit plus précisément ici de désignations vernaculaires de lectes avec lesquels les enquêtées déclarent être régulièrement en contact.

4. Si l'on considère que la notion de groupe de pairs se définit par : des interactions entre les membres d'un groupe, une influence mutuelle exercée par ses membres, l'intériorisation de normes et de valeurs communes susceptibles d'orienter les comportements, et l'appartenance reconnue par ses membres (Labov, 1978 [1972]), on est en effet fondé à considérer que l'on est en présence de deux groupes de pairs dans ce contexte institutionnel.

5. Les désignations qui suivent ont été relevées dans des propos d'élèves (EL) et/ou d'enseignants (ENS), dans divers contextes d'énonciation (en cours, en entretien, au cours de conversations informelles, etc.). En dehors de celles indiquées ici, bien d'autres ressources de catégorisation sociale, scolaire, ethnique, ou personnelle, sont sélectionnées par les jeunes filles 
« françaises/ratonnes » (ELs) «calmes/méchantes » (ENS) « élèves sérieux/groupe perturbateur »(ENS)... Ces couples de désignations - ainsi que de nombreux syntagmes non mentionnés ici - traduisent clairement une bi-polarisation des élèves au sein du groupe-classe et une dynamique relationnelle conflictuelle entre les deux groupes, ainsi qu'entre les « 13 » et des enseignants.

Sur le plan langagier, les différenciations entre les groupes d'élèves se manifestent dans la manière dont les sujets se représentent les langues, les normes, leur apprentissage, ainsi que dans des styles communicatifs (Kallmeyer, 2004) qui leur sont respectivement attribués. En effet, parmi les faisceaux de traits présentés tour à tour par les différents sujets comme caractéristiques des comportements, des normes et des attitudes des membres de chacun des groupes, la référence à des « façons de parler ${ }^{6}$ distinctes leur permet d'associer des ensembles de pratiques langagières et interactionnelles à leur groupe, et au groupe par rapport auquel ils/elles se positionnent.

Parmi les catégories vernaculaires les plus largement partagées par ces élèves pour décrire leur environnement sociolangagier, le «parler raton » est actualisé de manière récurrente. Si cette désignation axiologique n'est jamais utilisée par les jeunes filles pour qualifier leurs propres pratiques en français, elle sert néanmoins de pôle de référence commun aux élèves de la classe, qui l'actualisent afin de se situer, notamment au cours de nos entretiens.

\section{" ok $j(e)$ suis algérienne mais $j(e)$ suis pas ratonne "}

Comme la majorité des filles des deux groupes de la classe, Sonia et Angela, membres du groupe des 6, désignent Lamia, leader du groupe des 13, comme locutrice exemplaire du style communicatif qui caractérise, de leur point de vue, les comportements langagiers de la majorité des filles de l'autre groupe.

pour exprimer et configurer les relations inter- et intra-groupes. Par exemple, parmi les formes lexicales localement investies d'une valeur sociale spécifique dans le groupe des 13, «narvaline », « tunard », « ratonne », « crevard/ e », etc., peuvent constituer en contexte, selon les réglages de sens entre les participants, des marques de connivence, de solidarité ou de divergence à l'intérieur du groupe.

6. Locution spontanément actualisée dans de nombreux discours d'enquêté(e)s.

7. Sonia, membre leader des « $6 »$. 
1. Sonia ben $\mathrm{j}(\mathrm{e})$ sais pas c'est une langue bizarre quoi $\mathrm{j}(\mathrm{e})$ sais pas quand $\mathrm{j}(\mathrm{e})$ parle moi / et quand par exemple Lamia va vous parler / d(é)jà vous allez sentir : la différence $^{8}$

2. Angela mais non pa(r)ce que elle elle agresse les gens / quand elle parle

3. Enq donc toi tu trouves que c'est agressif

4. Angela ouais / c'est plus agressif le raton // pour moi / par exemple Sonia elle est posée par exemple vous emmenez L+ [= Lamia] elle va vite s'énerver [...] e(ll)e va vite s'énerver // quand [t]+ /// puis les ratons c'est c'est quelqu'un qui veut pas $\mathbf{s}(\mathbf{e})$ faire laisser marcher sur les pieds [...]

5. Sonia elle a une façon $\mathbf{d}(\mathbf{e})$ parler différente à nous $[\ldots]$ peut-être par rapport à son quartier

6. Angela Voilà

$[\ldots]$

7. Sonia mais en fait si vous voulez / nous on a l'impression tu vois / pour elles c'est j(e) sais pas c'est un peu ratonnes / et puis françaises /// j'ai l'impression qu(e) ça fait ça [...] ouais voilà / en plus / y a j'ai l'impression qu(e) ça faisait clan ratons et puis clan français

8. Enq et donc vous vous étiez mis dans le+<

9. Angela camp des Français

10. Enq le camp des Français

11. Angela ben en fait si vous voulez moi je suis pas vraiment ratonne enfin je+ ok $\mathrm{j}(\mathrm{e})$ suis algérienne mais $\mathbf{j}(\mathrm{e})$ suis pas ratonne $[\ldots]$

Les deux adolescentes accomplissent ici un effort de distinction qui les conduit à se différencier des pratiques communicatives qu'elles

8. Dans les transcriptions : notation entre ( ) de phonèmes ou segments non réalisés et dont la réalisation peut être variable (par exemple le « e muet ») ; la réalisation des schwa ( $e$ muets) est indiquée par E majuscule. 
attribuent à Lamia et à son groupe $(1 ; 5)$. Cette prise de distance s'accompagne d'une définition largement partagée dans la classe de la catégorie stigmatisée des «ratonnes ». La schématisation discursive de présentation du style de parole «raton » en direction de l'enquêtrice $^{9}$ repose ainsi sur l'identification d'une fonction identitaire réactive, agressive et défensive $(2 ; 4)$ liée à des modes de socialisation de « quartier » (5). On devine par ailleurs à partir de cet extrait que les rapports d'inclusion/exclusion aux groupes reposent fréquemment sur l'actualisation de catégories ethniques (tours 7 et 9) qui renvoient à des différences sociales et à des postures divergentes vis-à-vis de l'institution et du travail scolaires. En effet, les processus qui conduisent à la formation de deux groupes ethnicisés opèrent moins sur la base d'origines familiales étrangères que sur celle de comportements langagiers associés à des perspectives sociales et scolaires. C'est ce dont témoigne la déclaration de Sonia, en clôture de séquence : «ok $j(e)$ suis algérienne mais $j(e)$ suis pas ratonne » (11). Fille de primomigrants algériens, Sonia ne semble en effet n'avoir ici d'autre choix que cette forme de concession apparemment ambigüe, mais en réalité parfaitement cohérente avec la stratégie identitaire d'assimilation au majoritaire (Leonetti-Taboada, 1991) qui se manifeste dans la plupart de ses comportements ${ }^{10}$, y compris langagiers.

\section{"pour la moindre chose ça y est $j(e)$ suis une ratonne $" 1$}

Nombre de discours - dont certains circulent largement dans les sphères médiatiques, politiques, scientifiques et éducatives - attribuent à des descendants de migrants maghrébins de milieux économiquement défavorisés un répertoire verbal incomplet, lacunaire, renvoyant ces jeunes locuteurs à une identité de monostyles, doublée de celle de semilingues.

Des injonctions normatives visant à prévenir ou contrecarrer des comportements contre-normés sont parfois au principe de stratégies

9. Elle-même probablement catégorisée comme française et adulte.

10. Le fait qu'elle préfère se faire appeler par son second prénom, «Sonia », plutôt que «Soraya », premier prénom dans son état civil, constitue un autre élément au service de ce type de stratégie identitaire ; «Sonia » présentant moins de marques d'une appartenance catégorielle ethnique que « Soraya ».

11. Lamia, membre leader des « $13 »$. 
d'assimilation au majoritaire telle que celle visiblement adoptée par Sonia. Mais elles peuvent également favoriser des stratégies de surenchère identitaire (Leonetti-Taboada, 1991). Des processus interactifs de pratiques-assignations-intériorisations sont d'ailleurs sans doute à même d'éclairer l'essentialisation monostyle d'élèves telles que Lamia et d'autres membres du groupe des 13. Cette focalisation sur le déviant est tout aussi susceptible de masquer l'étendue de leurs ressources langagières.

Généralement caractérisée par les membres du groupe des « 6 » comme représentante emblématique de la « ratonne », Lamia exprime dans l'extrait d'entretien suivant ${ }^{12}$ son refus d'être assignée et réduite à cette identité stigmatisée.

1. Lamia [...] mais ouais mais main(te)nant mais pour la moindre chose ça $\mathbf{y}$ est $\mathbf{j}(\mathrm{e})$ suis une ratonne / $\mathrm{j}(\mathrm{e})$ vais dire « la vie $\mathrm{d}(\mathrm{e})$ ma mère » ça $\mathrm{y}$ est $<\mathrm{j}(\mathrm{e})$ suis/c'est $>$ une ratonne / $d(e)$ toute façon $j(e)$ vais dire « ma parole c'est vrai » et hop / pour pas jurer $\mathrm{j}(\mathrm{e})$ vais dire «ma parole » $\mathrm{i}(\mathrm{l})$ va quand même dire que $\mathrm{j}(\mathrm{e})$ suis une ratonne / la moindre ch+ et lui i(l) veut+ attends i(l) faut pas abuser / j(e) vais pas parler aussi du soutenu aussi / « oh excusEmoi / tu peux mE passer $1 E$ verre s'il tE plaît car $\mathrm{j}$ 'en ai besoin pour boire un $\mathrm{v}+$ un coup » (imite un style 'soutenu' : débit plus lent, voix légèrement plus aigüe, moins de «e» muets, lexique) faut pas abuser aussi hein

2. Enq pourquoi pourquoi tu tu voudrais pas parler comme ça

3. Lamia $j(e)$ vais pas parler le langage soutenu $j(e)$ parle normalement / euh correct / normal / pas b(e)soin $\mathrm{de}+\mathrm{j}(\mathrm{e})$ vais pas dire « $\mathrm{OH} /$ donne-moi ça sinon $\mathrm{j}(\mathrm{e})$ vais $\mathrm{t}(\mathrm{e})$ marave » (imitation contretype de 'soutenu': «e » muets, cri contenu, lexique) (rires) /// j(e) parle normalement :: ouais : et lui : <à> la moindre chose $\mathrm{i}(\mathrm{l})$ dit ben voilà « une ratonne »

12. En présence du chercheur mais également de sa meilleure amie Sana. 
En cherchant à ne pas se laisser enfermer dans des logiques d'allégeance dichotomiques ( $\mathrm{ou} . . . \mathrm{ou} » \ldots$ «ni... ni »), Lamia met ici en œuvre une stratégie linguistique et identitaire «sur le fil du rasoir» (Woods, 1990). Tout en soulignant un rejet de la pression normative exercée par un de ses frères, elle propose une description de ce qu'elle entend par «normal », « soutenu » et « ratonne», en rapportant cette description à des situations familiales qui relèvent de la sphère privée. Dans cette activité de catégorisation de styles, c'est principalement l'imitation qui lui sert de procédé différentiel pour construire deux contretypes langagiers («soutenu» et «ratonne») et affirmer pour ce qui concerne sa propre façon de parler, un positionnement médian qu'elle qualifie de «normal».

Elle manifeste ainsi une compétence de variation stylistique (Labov, 1978 [1972]) dont la séquence interactionnelle suivante suggère une amplitude rarement perçue (et/ou perceptible ?) au sein de l'espace scolaire.

\section{« excusez-moi madame »...}

Il s'agit d'un extrait d'une interview que Lamia réalise et enregistre sur un marché urbain, dans le cadre d'une activité scolaire en cours de français. Celle-ci consiste dans la réalisation, par les élèves, d'une enquête sur le plurilinguisme de l'agglomération grenobloise.

La principale motivation didactique pour la conception et la mise en œuvre de cette activité « hors les murs » du lycée reposait sur la volonté de favoriser des possibilités de rencontres et d'échanges, en sortant de l'établissement scolaire, lieu d'assignations négatives mutuelles entre enseignants et élèves, et de postures anti-scolaires pour certains élèves.

La séquence se déroule donc à l'extérieur du lycée, et Lamia aborde une inconnue d'une soixantaine d'années (L1 dans la transcription).

1. Lamia (minidisque visible dans les mains) excusez-moi madame

2. L1 oui $[\ldots]$

3. Lamia (ralentissement $d u$ débit) on est dans le lycée André Gajart // et euh on $\mathrm{f}+$ on pose des questions aux : clients aux gens qui travaillent dans ce marché 
4. L1 oui

5. Lamia et on voudrait savoir si on <pourrait/pouvait> vous poser des questions / ça s(e)rait po[ssible'

6. L1 euh oui / si vous voulez

7. Lamia on voudrait savoir : combien d(e) langues vous utilisez dans votre vie / des langues

8. L1 combien d(e) langues'

9. Lamia si le français l'italien ::

10. L1 oui français et: je utilise une langue que vous connaissez pas

11. Lamia vous pouvez la dire s' $i<1>$-vous-plaît

12. L1 letton $(\approx[$ letõ] $)[\ldots]$ ça c'est des Pays Baltes / qui est au nord $[\ldots]$

13. Lamia et : cette langue quand+ vous vous+ / euh attendez excusez-moi / vous connaissez beaucoup de gens qui la parlent'

14. L1 euh : il y a quelques familles qui habitent ici qui parlent aussi /// notre langue [...] qui parlent la langue lettone / nous sommes de Lettonie

15. Lamia Lettonie

16. L1 oui / Riga / avec les la la la la / le+ la ville principale est Riga

17. Lamia ah : c'est la ville :+ c'est la nationale en fait

18. L1 voilà

19. Lamia c'est la : c'est la CApitale

20. L1 c'est la capitale

21. Lamia voilà c'est la capitale

22. L1 eh oui

23. Lamia eh ben merci bien madame / passez une très bonne journée / au r(e)voir

24. L1 oui / merci / au r(e)voir 
Cette séquence interactionnelle met en évidence une capacité chez Lamia à jouer sur différents registres sociocommunicatifs et identitaires. Lamia porte en effet une forte attention à son discours, respecte les normes du genre interactionnel et discursif de l'interview (Gajo et Mondada, 2000), et manifeste un effort de convergence, d'ajustement et de co-énonciation avec la personne interviewée. Une comparaison avec la séquence précédente permet encore de souligner des variations importantes, notamment aux niveaux de la hauteur de voix, du rythme et du débit.

Comme Lamia, d'autres élèves du groupe des 13 s'impliquent activement dans la réalisation de cette enquête sur le plurilinguisme urbain. Elles puisent largement dans leur répertoire et mettent en œuvre des stratégies communicatives qui leur donnent corps en tant que locutrices, actrices sociales, et apprenantes à part entière. Pour certaines d'entre elles - et c'est le cas de Lamia - leur implication et les capacités qu'elles manifestent lors de cette activité tranchent singulièrement avec leur image scolaire d'élèves déviantes et de locutrices incompétentes.

Lors du bilan collectif de cette enquête en classe, plusieurs petites « réussites interactionnelles » donneront lieu à des manifestations d'une certaine fierté. Ces sentiments de réussites, collectifs et/ou individuels, apparaissent d'autant plus significatifs que les rencontres et les échanges qui les ont générés s'instituent, du fait de leur thématisation en classe, en objets de réflexion dans le cadre d'une activité scolaire.

Sans apaiser l'ensemble des tensions qui caractérisent les relations au sein de la classe, le déroulement de cette séquence didactique contredit l'apparente fermeture identitaire des groupes et de leurs membres. Les brèches interculturelles ainsi entrouvertes semblent en effet propices à un processus de défigement de certaines postures, dont celle, habituellement plus normative, de l'enseignant.

\section{En guise de conclusion. L'ouverture à la pluralité linguistique en formation d'enseignants : justifications, résistances et perspectives}

Dans le cadre de cette recherche-action, des sessions de formation visaient à engager une réflexion avec les enseignants qui permettrait de déboucher sur des pistes concrètes de prise en compte de la pluralité et de la variabilité linguistiques et culturelles.

Or, la tonalité normative qui a prévalu lors des sessions de formation, ainsi que la prégnance de certains discours sur les « jeunes des 
banlieues », ont fait bien souvent écran au débat que l'on cherchait à instaurer. Dans ce contexte, des postures radicales se sont affirmées dans des échanges où transparaissaient de multiples rapports de force et où les problèmes étaient le plus souvent posés dans des termes empruntés à la rhétorique de "l'intégration » et du refus d'intégration de certains élèves. Deux tendances, l'une consistant à adopter une posture de «neutralité affective » vis-à-vis des différences linguistiques et culturelles (van Zanten, 2001, p. 333) et l'autre relevant d'une vision essentialiste des différences, ont sans doute représenté des freins puissants au travail entrepris dans ce volet de la coopération avec le lycée. Les résistances paraissaient s'ancrer dans des réalités subjectives très fortes dans l'espace scolaire, par exemple lorsque des enseignants évoquaient la «pauvreté » de langage de certains élèves, ou les «handicaps » générés par des conditions de socialisation familiale plurilingue.

Comme le soulignent Georges Lüdi et Bernard Py (2003 [1986]), l'une des plus grandes difficultés dans la formation des enseignants réside dans la stabilité de ce type de représentations ${ }^{13}$. Or, si l'on souhaite en faire des objets de réflexion en formation, il semble nécessaire de rechercher des voies qui permettent d'amorcer des déplacements de regard sur ces questions.

Ces déplacements de regard supposent d'abord, selon nous, une intégration particulièrement marquée dans le paradigme des démarches dites «interculturelles». La finalité des démarches ainsi nommées n'étant pas de reconnaître à des élèves des «langues-cultures » différentes, au risque de figer les différences et d'y enfermer les élèves, mais bien plutôt au contraire de travailler sur la diversité des identités sociales, y compris celles des professionnels de l'éducation, dans un processus d'élaboration de significations partagées, c'est-à-dire d'une culture langagière commune non figée et non fétichisée (Billiez et Lambert, 2008).

Ce cadre implique qu'il faille aborder en formation des thèmes qui dérangent. Les blocages liés aux représentations des langues, de la communication verbale et des missions de l'institution scolaire en matière d'éducation linguistique, mais d'autres encore, de nature plus structurelle ou institutionnelle, ont souvent limité les effets de ces

13. Représentations sans doute d'autant plus stables qu'elles peuvent se voir confortées par les discours (scientifiques et/ou de vulgarisation) de certains linguistes. 
sessions de stages. Les bilans des formations et des expérimentations dans les classes ont même parfois eu tendance à susciter un sentiment d'échec. L'une des raisons est que nous n'avions sans doute pas saisi, de prime abord, que la pédagogie du « détour », fondamentale dans les démarches d'ouverture à la pluralité linguistique et culturelle destinées aux élèves, aurait dû davantage guider la conception des contenus de formation à l'intention d'enseignants eux-mêmes concernés personnellement par les questions de plurilinguisme et de migrations ${ }^{14}$.

Ces quelques éléments esquissés plaident également, et tout particulièrement, en faveur d'une intégration accrue de contenus sociolinguistiques dans les formations initiales des enseignants (Dabène, 1994 ; Perregaux, 2007) ainsi que celle d'activités d'ouverture à la pluralité linguistique et culturelle (Billiez, 1997 et 2005 ; Allemann-Ghionda et al., 1999).

Une reconnaissance de la pluralité et de la variabilité linguistiques, dans tous les volets du système éducatif, ne peut en effet advenir qu'à condition que la diversité soit réellement inscrite au cœur du projet éducatif (Coste, 2005, p. 401)...

\section{RÉFÉRENCES BIBLIOGRAPHIQUES}

Alleman-Ghionda C., de Goumoëns C. et Perregaux C. (1999) : Pluralité linguistique et culturelle dans la formation des enseignants, Éditions Universitaires Fribourg, Suisse.

BILLIEZ J. (1997) : Bilinguisme, variation, immigration : regards sociolinguistiques, vol. 1 et 2 , Thèse d'habilitation à diriger des recherches, Université Stendhal-Grenoble 3.

14. Le type de trajectoires sur lesquelles la plupart de ces enseignants plurilingues s'étaient engagés longtemps auparavant était celui d'une ascension sociale favorisée par un processus d'assimilation linguistique et culturelle « à la française ». Partant, ce que nous leur proposions d'entreprendre avec leurs élèves, à savoir accueillir, au moins symboliquement, l'ensemble des ressources linguistiques des élèves, supposait aussi l'accueil de celles héritées des migrations. Or, cette perspective entrait en contradiction avec les convictions que ces enseignants s'étaient forgées, à l'aune de leur propre expérience, quant aux missions de l'école républicaine en matière d'éducation linguistique. 
Billiez J. (dir.) (1998) : De la didactique des langues à la didactique du plurilinguisme. Hommage à Louise Dabène, CDL-LIDILEM, Université Stendhal - Grenoble 3.

Billiez J. (2005), « Répertoires et parlers plurilingues. Déplacements à opérer et pistes à parcourir à l'école », Tupin et Wharton (eds), Du plurilinguisme à l'école, Bern, Peter Lang, p. 323-339.

Billiez J. et LAmbert P. (2008) : «Autour de "savoirs" sur les langues dans une classe de seconde professionnelle », dans M. Candelier, G. Ioannitou, D. Omer et M. T. Vasseur (dir.), Conscience du plurilinguisme : pratiques, représentations et interventions, Presses Universitaires de Rennes, p. 79-91.

Coste D. (2005) : «Éléments pour une construction utopique nécessaire », Tupin et Wharton (eds), Du plurilinguisme à l'école, Bern, Peter Lang, p. 401-416.

DABÈne L. (1994) : Repères sociolinguistiques pour l'enseignement des langues, Paris, Hachette.

Gajo L. et Mondada L. (2000): Interactions et acquisitions en contexte. Modes d'appropriation de compétences discursives plurilingues par de jeunes immigrés, Fribourg, Éditions Universitaires.

Kallmeyer W. (2004) : «Variation multilingue et styles sociaux communicatifs. L'exemple de jeunes migrants turcs en Allemagne », Langage et Société, 109, p. 75-93.

LABOv W. (1978 [1972]) : Le parler ordinaire, Paris, Éditions de Minuit.

LAMBERT P. (2005) : Les répertoires plurilectaux de jeunes filles d'un lycée professionnel. Une approche sociolinguistique ethnographique, Thèse de doctorat de sciences du langage sous la direction de J. Billiez, Université Stendhal - Grenoble 3.

Leconte F. et Delabarre E. (2003) : «L'évaluation de l'hétérogénéité linguistique par les enseignants de collège », dans Caitucoli (dir.), Situations d'hétérogénéité linguistique en milieu scolaire, Rouen, Publications de 1'Université de Rouen, p. 59-81.

LeOnetti-Taboada I. (1991) : « Stratégies identitaires et minorités », dans Migrants formation, $\mathrm{n}^{\circ} 86$, p. 54-73.

LÜDI G. et Py B. (2003 [1986]) : Être bilingue, Bern, Peter Lang [3éd.].

Perregaux C. (2007) : «Avec le biographique, développer l'intérêt pour la complexité des répertoires langagiers dans la formation et la recherche », dans M. Verdelhan-Bourgade (dir.), Le français langue seconde : un concept et des pratiques en évolution, Bruxelles, De Boeck, p. 143-146.

Van Zanten A. (2001) : L'école de la périphérie. Scolarité et ségrégation en banlieue, Paris, PUF (coll. Le lien social).

Woods P. (1990) : L'ethnographie de l'école, Paris, Armand Colin. 
